\title{
The Utility of Endovaginal Ultrasound in the Design of a Sacrospinous Ligament Anchoring Device in Patients with and without Pelvic Organ Prolapse
}

\author{
Jonia Alshiek',2, Qi Wei ${ }^{3}$, Lieschen H. Quiroz ${ }^{4}$, Mikio A. Nihira5, Menahem Neuman6, \\ S. Abbas Shobeiri ${ }^{1,3 *}$ \\ ${ }^{1}$ Department of Obstetrics \& Gynecology, INOVA Women's Hospital, Falls Church, USA \\ ${ }^{2}$ Department of Obstetrics and Gynecology, Hillel Yafe Medical Center, Hadera, Israel \\ ${ }^{3}$ Department of Bioengineering, George Mason University, Fairfax, USA \\ ${ }^{4}$ Department of Obstetrics \& Gynecology, The University of Oklahoma Health Sciences Center, Oklahoma City, USA \\ ${ }^{5}$ Department of Obstetrics \& Gynecology, The University of California, Riverside, USA \\ ${ }^{6}$ Urogynecology, Women Health Division, Galilee MC, Nahariya, Faculty of Medicine, Bar Ilan University, Ramat Gan, Israel \\ Email: *Abbas.Shobeiri@inova.org
}

How to cite this paper: Alshiek, J., Wei, Q., Quiroz, L.H., Nihira, M.A., Neuman, M. and Abbas Shobeiri. S. (2019) The Utility of Endovaginal Ultrasound in the Design of a Sacrospinous Ligament Anchoring Device in Patients with and without Pelvic Organ Prolapse Open Journal of Obstetrics and Gynecology, 9, 1103-1113.

https://doi.org/10.4236/ojog.2019.98107

Received: June 9, 2019

Accepted: August 6, 2019

Published: August 9, 2019

Copyright $\odot 2019$ by author(s) and Scientific Research Publishing Inc. This work is licensed under the Creative Commons Attribution International License (CC BY 4.0).

http://creativecommons.org/licenses/by/4.0/

\section{(c) (i) Open Access}

\begin{abstract}
Objective: To determine whether endovaginal ultrasound is a reliable measure in visualization of the sacrospinous ligament among women with prolapse versus women without prolapse, and thus might be clinically applicable in the design of an ultrasound-guided device for performing sacrospinous ligament anchor placement as a treatment for pelvic organ prolapse. Methods: In the first phase of this study we performed a sacrospinous anchor placement in four normal fresh-frozen female pelves. Afterwards, an endovaginal ultrasound was performed to visualize the anchor localization which was validated by dissection of the cadaveric pelves. In the second phase of the study: two groups of volunteer females with and without pelvic organ prolapsed (POP-group, vs NON-POP group) were evaluated by endovaginal ultrasound to localize the sacrospinous ligament. Results: Cadaveric dissection demonstrated accurate anchor placement into the $8 / 8$ sacrospinous ligament. We performed endovaginal ultrasound in a total of $17 \mathrm{~N}-\mathrm{POP}$ and 10 (POP) patients. Among the N-POP group, the right and left ischial spines were visible in 6/17 (35\%) and (6/17) 35\% vs 0/10 (0\%) for both right and left sides in POP group $(\mathrm{p}=0.008)$. The right sacrospinous ligament was visualized in $4 / 17(23 \%) \mathrm{N}$-POP subjects and 0/10 POP subjects $(\mathrm{p}=0.27)$ and the left sacrospinous ligament was visualized in 7/17 (41\%) N-POP subjects and 2/10 POP subjects $(\mathrm{p}=0.48)$. Conclusions: Sacrospinous ligament and the ischial
\end{abstract}


spines couldn't be reliably visualized among women with or without pelvic organ prolapse using endovaginal ultrasound, although the structures are visualized more in some of the non-prolapsed women. The sacrospinous anchoring device demonstrated accurate placement by cadaveric dissections.

\section{Keywords}

Sacrospinous Ligament, Pelvic Organ Prolapse, Endovaginal Ultrasound

\section{Introduction}

Pelvic Organ Prolapse (POP) occurs in up to $50 \%$ of women based on vaginal exam, and the lifetime prevalence of symptoms is $3 \%-6 \%$ [1]. Based on a US claims and encounters database, the estimated lifetime risk of surgery for pelvic organ prolapse in women is $13 \%$ by the age of 80 [2]. The etiology is multifactorial, and the annual estimated cost of POP is more than $\$ 1$ billion in the US alone [3]. The treatment of POP could be conservative [4] or surgical [1]. The treatment is chosen based on the prolapse stage based on Pelvic Organ prolapse quantification score [5], patient age, patient's Gynecological/Obstetrical, medical background, and the training and experience of the surgeon. Many different procedures have been developed [3] [6] [7]. One of the oldest, important and popular surgical techniques of POP repair is sacrospinous ligament (SSL) fixation in which the apical portion of the vagina is anchored to the sacrospinous ligament [8] [9].

In 1892 Zweifel described a parasacral fixation of the vaginal vault to the uterosacral ligament [10]. In 1927, Miller introduced a transvaginal technique for vaginal vault fixation near the base of the sacro-uterine ligament, approximately 11/2 inches below the promontory of the sacrum [11]. In contrast, the abdominal approach has been emphasized by other study groups, making a laparotomy necessary [12] [13]. In 1951 Amreich first published a case report of sacrospinous ligament fixation by selecting a parasacral approach after resection of the coccygeal bone [14]. Sederl and Richter fixed the vaginal vault at the sacrospinous ligament alone by the vaginal route and showed long-term follow-up data of this operation [15] [16] [17]. In 1971 Nichols and Randall introduced the sacrospinous ligament fixation in the United States [18] [19] [20]. Long-term follow up data for recurrence are rarely presented in the literature ranging between $1 \%$ and $8 \%$ [16] [21]. Sacrospinous hysteropexy on the other hand has had a 2.3\% failure for the descensus of uterus, and a 35\% cystocele recurrence which tend to be asymptomatic [22]. Generally, a sacrospinous ligament suspension required dissection of the paravaginal space to identify the SSL and has been associated with risk of bleeding, damage nerves in the area, and/or compromise the ureter [9] [23] [24]. Sacrospinous ligament is a durable point of attachment for sutures, although there is considerable variation in sacrospinous ligament anatomy [25] [26]. The maximum force needed to break the sacrospinous liga- 
ment is $209 \mathrm{~N}\left(1 \mathrm{~N}=\right.$ mass $\left.\times 9.81 \mathrm{~m} / \mathrm{s}^{2}\right)$ versus $435 \mathrm{~N}$ for the sacrotuberous ligament. By comparison, only $50 \mathrm{~N}$ force is needed to break a single strand of untied 0 polypropylene suture. The sacrospinous ligaments generally fail near the mid-substance, whereas $75 \%$ of the sacrotuberous ligaments brake near the ischial tuberosity attachment [27].

A novel device NeuGuide ${ }^{\mathrm{Tm}}$ (POP Medical Solutions, Tel Aviv, Israel) was designed to circumvent the complications associated with sacrospinous ligament space dissection. Although placement of an anchor in the sacrospinous ligament has previously been reported [28] [29] [30], the NeuGuide ${ }^{\mathrm{TM}}$ device is aimed at performing sacrospinous hysteropexies. This device is currently undergoing trials for clinical applications. The NeuGuide ${ }^{\mathrm{T} \mu}$ anchor's mean pull-out force from the sacrospinous ligament was $35.68 \pm 9.28$ in a previous study. None of the measured forces was below $20 \mathrm{~N}$, which was the predefined lower $95 \%$ tolerance interval for the pullout force [31].

This procedure was designed to allow surgeons to repair uterovaginal prolapse in a minimally invasive manner to provide lasting suspension of the cervix to the SSL and spare the uterus from a hysterectomy. Localization of the suture on the SSL is the challenging part of the procedure; therefore, visualization of the SSL by ultrasound prior to the suturing might be useful.

To our knowledge there are no reports of feasibility of using US as a reliable measure in localization of sacrospinous ligament or for an US guided minimally invasive procedures. Transgluteal visualization of the ischial spines for SSL and sacrotuberous ligament (STL) injection of local anesthetic and steroid to the pudendal nerve in patients with pudendal neuralgia has been reported [32] [33].

In this study we aimed to examine whether the vaginal $2 \mathrm{D}$ or $3 \mathrm{D}$ US is 1 ) reliable for the visualization of SSL in patients with and without pelvic organ prolapse, and 2) required for clinical use in order for the anchoring device to be placed accurately.

\section{Methods}

This was a pilot observational study that was initially approved by the Institutional Review Board at the University of Oklahoma Health Sciences Center and subsequently at Inova Fairfax Hospital for data analysis. All participants in the clinical part of the study signed appropriate research consent forms. The study was divided into two phases:phase I: cadaveric experiment designed to determine the accuracy of sacrospinous ligament anchoring using 2D/3D endovaginal US followed by cadaveric dissection, and phase II: a cross-sectional pilot study comparing the validity of $2 \mathrm{D} / 3 \mathrm{D}$ endovaginal US in visualization of SSL and IS among POP women versus N-POP women.

In the first phase of the study, pelves of four normal cadavers were prepared from unperfused/unfixed specimens with no history of POP. The cadavers were defrosted for 36 hours and brought to room temperature in an external bath. Then we performed a NeuGuide ${ }^{\mathrm{TM}}$ SSL anchor according to the device instructions [34] (Figure 1). Afterwards, 2D/3D endovaginal ultrasound (US) (BK 


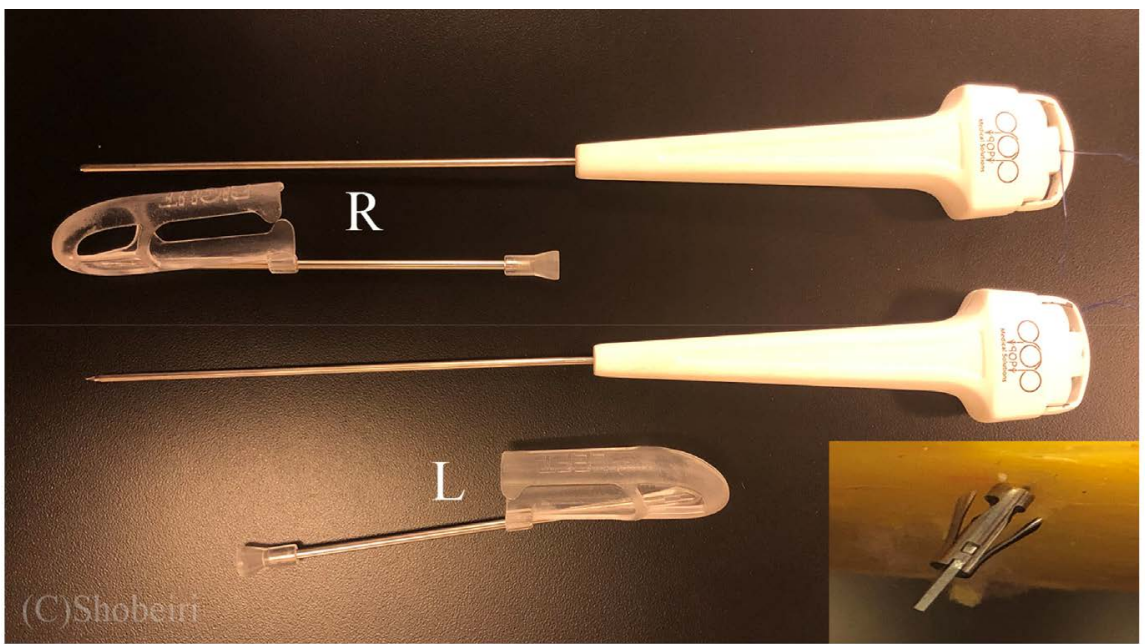

Figure 1. The NeuGuide ${ }^{\mathrm{Tm}}$ device; single use transvaginal pelvic floor repair system which enables delivery of a tissue anchor to the ligaments of the vaginal cavity. The anchors are pre-loaded inside the device shaft and are ready for use. A thimble is also supplied, as an accessory for the device, to be used as a guide channel for better handling of the NeuGuide $^{\mathrm{Tm}}$. The NeuGuide ${ }^{\mathrm{Tm}}$ device package contains three kits, each kit is supplied in a sterile blister pack. Each kit contains the following: 2 NeuGuide ${ }^{\mathrm{Tm}}$ devices with pre-loaded anchor and suture, 2 Thimbles (L \& R). R-Right; L-Left.

ultrasound, Analogic, Peabody, MA, USA) was performed using an endovaginal 8818 Triplane BK probe $(12-4 \mathrm{MHz})$ and an endovaginal $8838(12-4 \mathrm{MHz})$ probe to visualize the anchor localization. The anchor placement was validated by dissection of the cadaver pelves.

In the second phase of the study, two groups of women including a) non-prolapsed women with normal pelvic floors and no symptoms nor clinical evidence of POP (N-POP), and b) women with POP stage II and above (POP group); were evaluated by US in 2013-14 to localize the SSL and ischial Spine (IS). POP stage II and above was defined based on International Continence Society Pelvic Organ Prolapse Quantification System. Exclusion criteria included women under 18 years old, other concomitant pelvic floor abnormality, a history of a hysterectomy or a previous SSL intervention.

\section{Justification of the Sample Size and Data Analysis}

Since this was a pilot study, a convenient sample size was used to gather the necessary data points. Statistical analysis was performed on de-identified data using Stata/IC version 14.2 (StataCorp LLC, Texas). Visualization or non-visualization of the SSL and the other structures was analyzed as a dichotomous variable. A 95\% confidence interval (CI) was applied and a p-value of less than .05 was considered statistically significant. Two-sided t-test and a chi-squared test were used to analyze the data.

\section{Results}

Phase I: Four fresh-frozen female pelves were selected randomly following 
research agreement from The University of Oklahoma Cadaver program in 2013-2014. The four dissected pelves had no history of POP or pelvic floor surgery. The average age for the pelves was not available to us but the pelves are generally derived from older donors.

Firstly, we performed a NeuGuide ${ }^{\mathrm{Th}}$ sacrospinous anchoring for all of the four cadaveric pelves as described previously [34]. In summary, The NeuGuide ${ }^{\mathrm{Tx}}$ is designed to enable centro-apical pelvic floor support for the uterine cervix without need of either vaginal dissection or mesh implants in patients with a central compartment defect that need suspension. The NeuGuide ${ }^{\mathrm{m}}$ device is comprised of two main elements: an anchor unit and a delivery system. The delivery system enables the guidance, insertion and deployment of the anchor element. The device's anchor unit is designed as a sharp needle point made from Nitinol to enable piercing through the vaginal layers and the ligament. The anchor is deployed and placed with the use of an applicator. The anchor incorporates a Polypropylene suture at its distal end, which following its deployment enables fixation and the continuation of the surgical procedure as intended for the repair process. It has a thimble that is an accessory to the device and can be used as an introducer for better handling of the NeuGuide ${ }^{\mathrm{tm}}$. The anchor penetration diameter is $2.0 \mathrm{~mm}$. Once deployed (passed the SSL), the wings open to $4.0 \mathrm{~mm}$. The work channel length is $120 \mathrm{~mm}$ (this limits the anchor penetration depth beyond the ligament in order to avoid injury). The device shaft diameter is $2.5 \mathrm{~mm}$ and its length $285 \mathrm{~mm}$. The suture length is $70 \mathrm{~cm}$ and the work channel is designed to fit all sizes (self-adjusting). The applicator includes two concentric hollow shafts. The outer shaft constrains the anchor wings from being deployed. Once the button is pressed, the inner shaft pushes the anchor distally and allows the wings to deploy. The applicator is equipped with a safety latch that protects the button, to avoid undesired deployment [34]. During the procedure, The NeuGuide device is mounted on the right index finger, and introduced into the vaginal cavity. The right ischial spine and the SSL are palpated through the vaginal wall. The index finger is stabilized intimately to the mid SSL. The anchor is deployed, and adequate pull-out force is proven. A one $\mathrm{cm}$ longitudinal shallow and high mucosal incision is made at the posterior or anterior cervix. The anchor's suture is mounted on a virgin needle. The suture is inserted backwards through the vaginal wall at its entering point, passed under the vaginal epithelium, then through the cervical isthmus and out to the vaginal cavity again through the posterior or anterior cervix incision. The previous steps are repeated on the left side and the suture is tied appropriately. The small cervical incision is closed [34].

The cadaveric 2D/3D US performed following the procedure localized the anchor placement in the SSL in the four cadaveric pelves. The anchor placement was validated by dissection of the cadaveric pelves which did not reveal placement abnormalities or device malfunctions (Figure 2). All functional steps were performed successfully. After performing SSLF on cadavers, cadaveric dissection was performed by creating hemi-pelves for better SSL visualization after SSL 


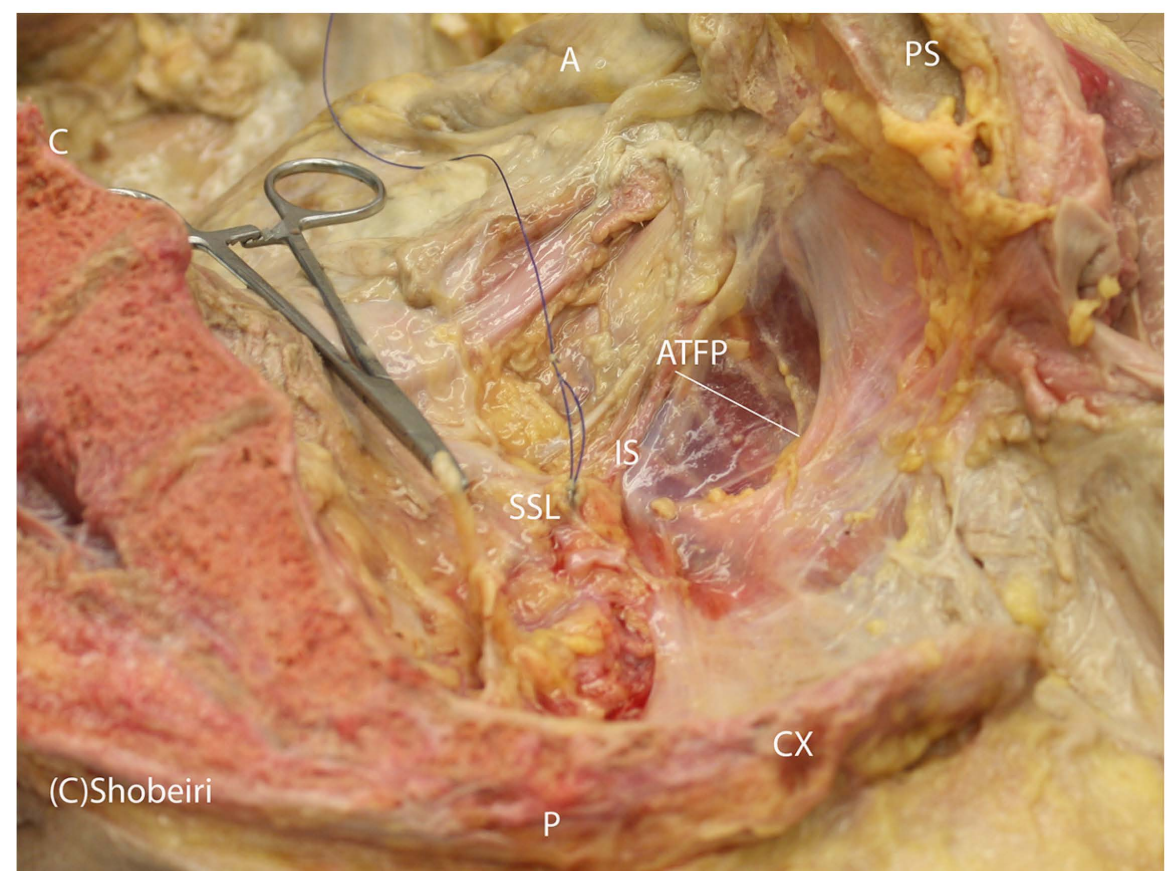

Figure 2. Cadaveric left hemipelvis with an intact anchor in the sacrospinous ligament. A-Anterior, ATFP-Arcus Tendinous Fascial Pelvis, C-Cephalad, CX-Coccyx, IS-Ischial Spine, P-Posterior, PS-Pubic symphysis, SSL-Sacrospinous ligament.

anchor placement. On all cadavers the SSL had been reached safely via a transvaginal approach for the NeuGuide ${ }^{\text {rx }}$ device guided by the accompanying thimble. No damage to the surrounding organs and tissues was demonstrated. We found that in all cases the actual position of the suture in relation to the mid SSL was correct; a safe distance was maintained from the rectum, bladder, and pudendal nerves and vessels; and the distance from the vagina or uterine cervix to the mid SSL was shortened in all cases.

Phase II: 17 volunteers without prolapse (N-POP) and 10 patients with POP were evaluated by endovaginal ultrasound to localize the SSL and IS (Figure 3).

We used two-sided t-test to assess the age and BMI for each group (Table 1). The N-POP group had a mean age of 62.7 (95\% CI: 59.0 - 66.4) while the POP group had a mean age of 63.8 (95\% CI: 58.7 - 68.9). The mean BMI of the N-POP group was 29.0 (95\% CI: 27.8 - 30.2) while that of the POP group was 28.3 (95\% CI: 25.7 - 31.0). Neither age nor BMI showed statistical significance between the two groups (p-value of 0.704 and 0.585 respectively).

Chi-squared test was used to analyze dichotomous data in the two groups. In general, the ischial spines were more visible in the N-POP group than the POP group $(\mathrm{p}=0.008)$. Among the N-POP group, we were able to visualize the right and left IS in 6/17 (35\%) and 6/17 (35\%) vs in POP group 0/10 (0\%) for both sides. No statistically significant difference was observed when the left and right ischial spines were compared separately ( $p=0.099$ for both left and right).

There was no statistically significant difference in the visibility of sacrospinous ligament between the two groups $(\mathrm{p}=0.12$ overall, $\mathrm{p}=0.48$ for the left and $\mathrm{p}=$ 


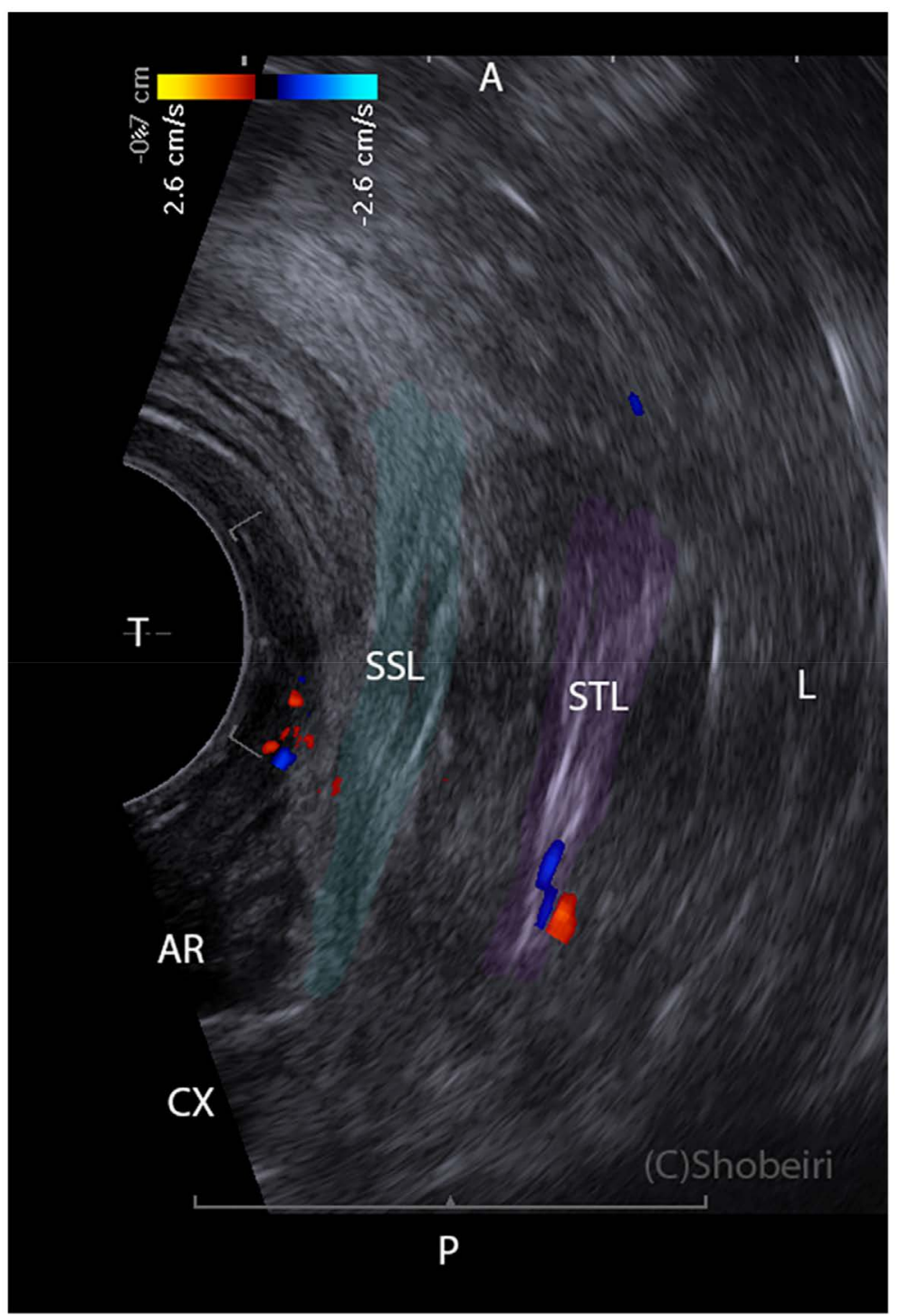

Figure 3. The ultrasound left axial view of sacrospinous ligament (in light blue), and the sacrotuberous ligament (in light purple) visible in a non-prolapsed subject with Doppler view of the blood vessels in the vicinity. A-Anterior, AR-Anorectum, CX-Coccyx, L-Left, P-Posterior, PS-Pubic symphysis, SSL-Sacrospinous ligament, STL-Sacrotuberous ligament, T-Vaginal Transducer.

Table 1. Mean \pm SD and 95\% confidence interval (CI) of age and BMI of the N-POP and POP groups. Two-sided t-test was performed to calculate the p-value. Mean age $62.7 \pm 7$ vs $63.8 \pm 7$; mean BMI $27.8 \pm 2$ vs $27.1 \pm 3.45$ in the N-POP vs POP respectively. $\mathrm{SD}$-standard deviation; CD-confidence interval; BMI-body mass index; POP-pelvic organ prolapse; N-POP-non-pelvic organ prolapse.

\begin{tabular}{cccccc}
\hline & \multicolumn{2}{c}{ N-POP $(n=17)$} & POP $(n=10)$ & \multicolumn{2}{c}{$p$-value } \\
\cline { 2 - 6 } & Mean $( \pm$ SD $)$ & $95 \%$ CI & Mean $( \pm$ SD $)$ & \multicolumn{2}{c}{$95 \%$ CI } \\
\hline Age & $62.7( \pm 7.2)$ & $59.0-66.4$ & $63.8( \pm 7.1)$ & $58.7-68.9$ & 0.704 \\
BMI & $29.0( \pm 2.4)$ & $27.8-30.2$ & $28.3( \pm 3.7)$ & $25.7-31.0$ & 0.585 \\
\hline
\end{tabular}


0.27 for the right). The SSL was visualized in N-POP group right 4/17 (23\%), left $7 / 17(41 \%)$ vs POP group right $0 / 10(0 \%)$, and 2/10 (20\%) (Table 2).

\section{Discussion}

The results of this study showed that the anchors were placed reliably in cadavers and that endovaginal ultrasonography was not required for the visualization of SSL or IS among POP or N-POP women. The findings of the study confirm that the SSL structures are visualized more in some N-POP women.

The main goal of this study was to determine whether 2D/3D Endovaginal Ultrasound necessary for visualization of the sacrospinous ligament among women with prolapse. Although an ultrasound-guided device for visualization of the sacrospinous ligament in non-prolapsed patient is feasible, ultrasound guided approach does not add value for the treatment of pelvic organ prolapse in this setting.

During the last two decades there have been many developments and innovations in the field of pelvic floor reconstructive surgery and imaging. Sacrospinous ligament suspension is one of the most effective treatments for POP. It could also be performed abdominally and laparoscopically. Based on the same rationale, a new device for the treatment of POP was innovated (NeuGuide ${ }^{\mathrm{Tm}}$ ) using the SSL as the anchor placement for the suspension of the uterus via a vaginal approach. The way an anchor is placed during the procedure is interesting since it requires no dissection and the anchor is placed through the vaginal epithelium. Initially our group explored perhaps a better way of precise anchor placement by using ultrasound visualization of the sacrospinous ligament. To our knowledge, there have been no studies for US or other imaging modality visualization for guidance of pelvic floor prolapse repair procedures.

Table 2. Summary of visibility of the ischial spines and sacrospinous ligament from endovaginal ultrasound images. Among the N-POP group, we were able to visualize the right and left Ischial spines in 6/17 (35\%) and (6/17) 35\% vs in POP group 0/10 (0\%) for both sides $(\mathrm{p}=0.008)$. The SSL were visualized in N-POP group right $4 / 17(23 \%)$, left $7 / 17(41 \%)$ vs POP group right $0 / 10(0 \%)(\mathrm{p}=0.27)$, and left $2 / 10(20 \%)(\mathrm{p}=0.48)$. POP-pelvic organ prolapse; N-POP-non-pelvic organ prolapse; SSL-sacrospinous ligament. Chi-squared test was performed to compute the $p$-value.

\begin{tabular}{cccc}
\hline & N-POP $(n=17)$ & POP $(n=10)$ & $p$-value \\
\hline Ischial spines & & $0(0 \%)$ & 0.099 \\
Left & $6(35 \%)$ & $0(0 \%)$ & 0.099 \\
Right & $6(35 \%)$ & & 0.008 \\
Left + Right & & & \\
Sacrospinous ligament & & $2(20 \%)$ & 0.48 \\
Left & $7(41 \%)$ & $0(0 \%)$ & 0.27 \\
Right & $4(23 \%)$ & & 0.127 \\
Left + Right & &
\end{tabular}


The limitations of the current pilot study are: the rather small sample size of dissected pelves (four) and the patients in phase II (17 POP, 10 N-POP). The recruitment was stopped based on interim analysis which showed accurate clinical placement of SSL anchor without the need for ultrasound guidance. Although one of the ultrasound observers was the principal investigator, and another was the inventor which may predispose to bias, this aspect was minimized by making the ultrasound images unidentifiable in respect to the volunteer's personal information.

Of note, the NeuGuide ${ }^{\text {tw }}$ device has been investigated outside of the United States and is undergoing trials in the US. In a study to evaluate the safety and short-term outcomes in 10 patients who had the procedure, the authors concluded there was no injury to the bladder, rectum, pudendal nerves, or major pelvic vessels. Patients were satisfied with the procedure and had favorable quality of life scores. The NeuGuide ${ }^{\text {rx }}$ device allowed rapid and safe introduction of a suspending suture through the sacrospinous ligament and made sacrospinous ligament fixation easy to perform, while avoiding dissection and mesh complications [34].

\section{Conclusion}

In conclusion, this sacrospinous ligament anchoring device for treatment of women with pelvic organ prolapse can be performed accurately without ultrasound guidance.

\section{Conflicts of Interest}

Professor Neuman is one of the Founders of POP Medical Solutions Ltd. ("POP") and a shareholder in POP. POP Medical Inc. Inc. is the US subsidiary of the Ltd. Company.

\section{References}

[1] Barber, M.D. and Maher, C. (2013) Epidemiology and Outcome Assessment of Pelvic Organ Prolapse. International Urogynecology Journal, 24, 1783-1790. https://doi.org/10.1007/s00192-013-2169-9

[2] Wu, J.M., Matthews, C.A., Conover, M.M., Pate, V. and Funk, M.J. (2014) Lifetime Risk of Stress Incontinence or Pelvic Organ Prolapse Surgery. Obstetrics and Gynecology, 123, 1201. https://doi.org/10.1097/AOG.0000000000000286

[3] Jelovsek, J.E., Maher, C. and Barber, M.D. (2007) Pelvic Organ Prolapse. The Lancet, 369, 1027-1038. https://doi.org/10.1016/S0140-6736(07)60462-0

[4] Chapple, C.R., Cruz, F., Deffieux, X., Milani, A.L., Arlandis, S., Artibani, W., et al. (2017) Consensus Statement of the European Urology Association and the European Urogynaecological Association on the Use of Implanted Materials for Treating Pelvic Organ Prolapse and Stress Urinary Incontinence. European Urology, 72, 424-431. https://doi.org/10.1016/j.eururo.2017.03.048

[5] Bump, R.C., Mattiasson, A., Bo, K., Brubaker, L.P., DeLancey, J.O., Klarskov, P., et al. (1996) The Standardization of Terminology of Female Pelvic Organ Prolapse and Pelvic Floor Dysfunction. American Journal of Obstetrics \& Gynecology, 175, 
10-17. https://doi.org/10.1016/S0002-9378(96)70243-0

[6] Geynisman-Tan, J. and Kenton, K. (2017) Surgical Updates in the Treatment of Pelvic Organ Prolapse. Rambam Maimonides Medical Journal, 8, e0017. https://doi.org/10.5041/RMMJ.10294

[7] Houman, J., Weinberger, J.M. and Eilber, K.S. (2017) Native Tissue Repairs for Pelvic Organ Prolapse. Current Urology Reports, 18, 6. https://doi.org/10.1007/s11934-017-0648-0

[8] Richter, K. (1968) The Surgical Anatomy of the Vaginaefixatio Sacrospinalis Vaginalis. A Contribution to the Surgical Treatment of Vaginal Blind Pouch Prolapse. Geburtshilfe und Frauenheilkunde, 28, 321.

[9] Shull, B.L., Bachofen, C., Coates, K.W. and Kuehl, T.J. (2000) A Transvaginal Approach to Repair of Apical and Other Associated Sites of Pelvic Organ Prolapse with Uterosacral Ligaments. American Journal of Obstetrics and Gynecology, 183, 1365-1374. https://doi.org/10.1067/mob.2000.110910

[10] Zweifel, P. (1892) Vorlesungen über klinische Gynäkologie. Hirschwald, Berlin.

[11] Miller, N.F. (1927) A New Method of Correcting Complete Inversion of the Vagina. Surgery, Gynecology \& Obstetrics, 44, 550-554.

[12] Yates, M. (1975) An Abdominal Approach to the Repair of Post-Hysterectomy Vaginal Inversion. BJOG, 82, 817-819. https://doi.org/10.1111/j.1471-0528.1975.tb00579.x

[13] Arthure, H.G. and Savage, D. (1957) Uterine Prolapse and Prolapse of the Vaginal Vault Treated by Sacral Hysteropexy. BJOG, 64, 355-360. https://doi.org/10.1111/j.1471-0528.1957.tb02652.x

[14] Amreich, J. (1951) Aetiologie und operation des scheidenstumpfprolapses. Wien Klin Wochenschr, 63, 74-77.

[15] Richter, K. (1967) Die operative Behandlung des prolabierten Scheidengrundes nach Uterusexstirpation. Ein Beitrag zur Vaginaefixatio sacrotuberalis nach Amreich. Geburtshilfe und Frauenheilkunde, 27, 941-954.

[16] Richter, K. and Albrich, W. (1981) Long-Term Results Following Fixation of the Vagina on the Sacrospinal Ligament by the Vaginal Route (Vaginaefixatio Sacrospinalis Vaginalis). American Journal of Obstetrics \& Gynecology, 141, 811-816. https://doi.org/10.1016/0002-9378(81)90709-2

[17] Sederl, J. (1958) Zur operation des prolapses der blind endigenden sheiden. Geburtshilfe Frauenheilkunde, 18, 824-828.

[18] Randall, C.L. and Nichols, D.H. (1971) Surgical Treatment of Vaginal Inversion. Obstetrics \& Gynecology, 38, 327-332.

[19] Nichols, D.H. (1982) Sacrospinous Fixation for Massive Eversion of the Vagina. American Journal of Obstetrics \& Gynecology, 142, 901-904. https://doi.org/10.1016/S0002-9378(16)32539-X

[20] Nichols, D. (1992) Enterocele and Massive Eversion of the Vagina. Te Linde's Operative Gynecology, 7, 855-885.

[21] Lantzsch, T., Goepel, C., Wolters, M., Koelbl, H. and Methfessel, H.D. (2001) Sacrospinous Ligament Fixation for Vaginal Vault Prolapse. Archives of Gynecology and Obstetrics, 265, 21-25. https://doi.org/10.1007/s004040000116

[22] Dietz, V., de Jong, J., Huisman, M., Schraffordt Koops, S., Heintz, P. and van der Vaart, H. (2007) The Effectiveness of the Sacrospinous Hysteropexy for the Primary Treatment of Uterovaginal Prolapse. International Urogynecology Journal, 18, 1271-1276. https://doi.org/10.1007/s00192-007-0336-6 
[23] Turner, L.C., Lavelle, E.S. and Shepherd, J.P. (2016) Comparison of Complications and Prolapse Recurrence between Laparoscopic and Vaginal Uterosacral Ligament Suspension for the Treatment of Vaginal Prolapse. International Urogynecology Journal, 27, 797-803. https://doi.org/10.1007/s00192-015-2897-0

[24] Kapoor, S., Sivanesan, K., Robertson, J.A., Veerasingham, M. and Kapoor, V. (2017) Sacrospinous Hysteropexy: Review and Meta-Analysis of Outcomes. International Urogynecology Journal, 28, 1285-1294. https://doi.org/10.1007/s00192-017-3291-x

[25] Vodušek, D.B. (2004) Anatomy and Neurocontrol of the Pelvic Floor. Digestion, 69, 87-92. https://doi.org/10.1159/000077874

[26] Woodley, S., Kennedy, E. and Mercer, S. (2005) Anatomy in Practice: The Sacrotuberous Ligament. New Zealand Journal of Physiotherapy, 33, 91-94.

[27] Shobeiri, S.A., Elkins, T.E. and Ka, T. (2000) Comparison of Sacrospinous Ligament, Sacrotuberous Ligament, and 0 Polypropylene Suture Tensile Strength. Journal of Pelvic Surgery, 6, 261-267.

[28] Shetty, S. and Kirkemo, A. (1997) Bilateral Bone Anchor Vaginal Vault Suspension: An Initial Report of a New Technique. Techniques in Urology, 3, 1-5.

[29] Goldberg, R.P., Tomezsko, J.E., Winkler, H.A., Koduri, S., Culligan, P.J. and Sand, P.K. (2001) Anterior or Posterior Sacrospinous Vaginal Vault Suspension: Long-Term Anatomic and Functional Evaluation. Obstetrics \& Gynecology, 98, 199-204. https://doi.org/10.1097/00006250-200108000-00004

[30] Giberti, C. (2001) Transvaginal Sacrospinous Colpopexy by Palpation-A New Minimally Invasive Procedure Using an Anchoring System. Urology, 57, 666-668. https://doi.org/10.1016/S0090-4295(00)01088-8

[31] Tsivian, M., Weintraub, A.Y., Neuman, M. and Tsivian, A. (2016) Introducing a True Minimally Invasive Meshless and Dissectionless Anchoring System for Pelvic Organ Prolapse Repair. International Urogynecology Journal, 27, 601-606. https://doi.org/10.1007/s00192-015-2875-6

[32] Rofaeel, A., Peng, P., Louis, I. and Chan, V. (2008) Feasibility of Real-Time Ultrasound for Pudendal Nerve Block in Patients with Chronic Perineal Pain. Regional Anesthesia and Pain Medicine, 33, 139-145. https://doi.org/10.1097/00115550-200803000-00009

[33] Peng, P. and Tumber, P.S. (2008) Ultrasound-Guided Interventional Procedures for Patients with Chronic Pelvic Pain-A Description of Techniques and Review of Literature. Pain Physician, 11, 215-224.

[34] Weintraub, A.Y., Zvi, M.B., Yohay, D., Neymeyer, J., Reuven, Y., Neuman, M., et al. (2017) Safety and Short Term Outcomes of a New Truly Minimally-Invasive Mesh-Less and Dissection-Less Anchoring System for Pelvic Organ Prolapse Apical Repair. International Brazilian Journal of Urology, 43, 533-539. https://doi.org/10.1590/s1677-5538.ibju.2016.0356 